\title{
ON SMOOTH BOUNDED MANIFOLDS ${ }^{1}$
}

\author{
GUDRUN KALMBACH
}

A noncompact smooth manifold $M$ is called a smooth bounded manifold if the following statement is true. There exist a compact subset $A \subset M$ and a smooth function $g$ defined on $M-A$ such that $g$ has no critical points on $M-A$ and such that $g\left(q_{i}\right) \rightarrow \infty$ for each sequence $q_{i} \in M$ without limit point on $M$.

We show that on each smooth bounded manifold there exists a smooth function $f$ which has only a finite number of nondegenerate critical points on $M$ and which has the property that $f\left(q_{i}\right) \rightarrow \infty$ for each sequence $q_{i} \in M$ without limit point on $M$.

Such a function will be called a critical finite function on $M$.

Together with the theorem of [5] it follows that to each strongly pseudoconvex Stein manifold with $C^{2}$-boundary (see $[4$, p. 262]) and with complex dimension $m$ there exists a finite $\mathrm{CW}$-complex $K \subset M$ of real dimension $m$ such that $K$ is a deformation retract of $M$. ( $K$ is the union of the finitely many descending bowls associated with some critical point of $f,[5]$ see $\$ 2$.)

In $\$ \S 1$ and 2 the manifold $M$ is a smooth bounded manifold and the function $g$ is given as above. $M$ shall have a Riemannian metric.

1. Motivation of " $M$ is smooth bounded." The theorem of this section shall give a motivation for the expression " $M$ is a smooth bounded manifold". It will not be used in $\$ \$ 2$ and 3 .

Let $C$ be a compact subset of $M$ with $A \subset \subset C$, i.e. the closure of $A$ is contained in $C$.

REMARK 1. There exists a number $e \in R$ such that $B_{b}=\bar{B}_{b}$ $=\{p \in M-C \mid g(p)=b\} \subset \subset M$ for $b \geqq e$.

Proof. Let $C=\bar{C} \subset \subset M$ such that $A \subset \subset C$. The function $g$ is defined on the boundary $\partial C$ of $C$ in $M$. Since $C$ is compact it follows that $g(p) \leqq d$ on $\partial C$ for some $d \in R$. Let $e>d$. Then for each set $B_{b}$ with $b \geqq e$ one has $B_{b} \cap \partial C=\varnothing$. Since $g\left(q_{i}\right) \rightarrow \infty$ for each sequence which has no limit point in $M$ it follows that $B_{b}=\bar{B}_{b} \subset \subset M$.

In the following, $C$ shall always be the subset of $M$ of Remark 1 . Let $e$ and $B_{b}$ be as in Remark 1 .

REMARK 2 . Let $b>e$ and $\psi(q)$ be the maximal orthogonal trajectory of $g$ through $q \in M-C$. Then there exists a parametrization

Received by the editors February 19, 1968.

1 The results are from the writer's thesis, Goettingen, 1966. This report was prepared under grant NSF GP 5610. 
$\left\{\psi_{t}(p) \mid t \in R\right\}$ of $\psi(p)$ with $p \in B_{b}$ such that $g\left(\psi_{t}(p)\right)=t+b$ if $t+b \geqq e$.

Proof. Since $g$ has no critical points in $\{p \in M-C \mid g(p) \geqq e\}$ one can use the theorem in $[7$, p. 13] to obtain in each compact set $\left\{p \in M-C \mid a_{i} \leqq g(p) \leqq a_{i+1}\right\}$ the result of Remark 2, where $a_{1}<a_{2}<a_{3}<\cdots$ with $\lim _{i \rightarrow \infty} a_{i}=\infty$. These parametrizations yield then the parametrization of Remark 2 .

There exists a smooth function $g^{\prime}(q)$ on $M-C$ such that $g^{\prime}(q)=0$ if $g(q) \geqq d$ and $g^{\prime}(q)=1$ if $g(q) \leqq b$ and $0 \leqq g^{\prime}(q) \leqq 1$ on $M-C$ and such that the functions $g^{\prime}$ and $g$ have the same level surfaces on $\{q \in M-C \mid b<g(q)<d\}$ (see [5, Remark 1]).

Below we will denote this function by $g_{b, d, o}(q)$ for $q \in M-C$.

Theorem 1. Let $M$ be a smooth bounded manifold. There then exists a compactification (see [2, p. 85]) $M^{\prime}=M \cup B$ of $M$ such that $B$ is a smooth manifold of dimension $n-1$ and $M^{\prime}$ is a smooth manifold with boundary $B$.

Proof. We show that there exists a diffeomorphism which maps $M$ on a proper subset $D \subset M$ such that the boundary $B^{\prime}$ of $D$ in $M$ is an $(n-1)$-dimensional manifold. Let $C, e$ and the parametrization of $\psi(p)$ be as above. Let $b>e$ and $d-b<1$ and $g^{\prime}(q)=1-g_{b, d, o}(q)$ if $q \in M-C$. Define $h(q)=h\left(\psi_{r}(p)\right)=\psi_{t}(p)$ with $p \in B_{b}$ and $0 \leqq r<1$ and $t=r /\left(1-g^{\prime}\left(\psi_{r}(p)\right) r\right)$. Let $h(q)=q$ if $g(q) \leqq b$ or $q \in C$. Then $h$ is a diffeomorphism of $D=\{q \in M \mid q \in C$ or $g(q)<b+1\}$ onto $M$. Since $B^{\prime}=\{q \in M-C \mid g(q)=b+1\}$ is an $(n-1)$-dimensional smooth manifold and $D \cup B^{\prime}$ is a smooth manifold with boundary $B^{\prime}$, Theorem 1 is true.

2. Critical finite functions on a smooth bounded manifold. Let $M \subset R^{k}$ be differentiably embedded in $R^{k}$. Let $x \in R^{k}$ be a fixed point and $L_{x}(p)=|p-x|^{2}$ the euclidian distance between $p$ and $x$ for $p \in M$. The function $L_{x}$ is smooth on $M$.

The following two propositions are from $[7$, p. 36f].

Proposition 1. For almost all $x \in R^{k}$ (all but a set of measure 0 ) the function $L_{x}$ has no degenerate critical points.

Proposition 2. Let $h$ be a smooth function on $M$ such that $\{p \in M \mid h(p) \leqq c\} \subset \subset M$ for each $c \in R$. Let $K \subset M$ be a compact set. Then $h$ can be uniformly approximated on $K$ by a smooth function $f$ on $M$ which has no degenerate critical points. Furthermore $f$ can be chosen such that the $i$ th derivative of $f$ on the compact set $K$ uniformly approximates the corresponding derivative of $h$ for $i=1,2$. One has $f=c \cdot L_{x}+d$ for some $x \in R^{k}$ and some constants $c>0$ and $d$. 
Theorem 2. Let $M$ be a smooth bounded manifold. Then there is a critical finite function $f^{\prime}$ on $M$.

Proof. Let $a>a^{\prime} \geqq e$ and $g_{1}(p)=g_{a^{\prime}, a, g}(p)$ for $p \in M-C$ (see Remark 1). Define $g^{+}(p)=\left(1-g_{1}(p)\right) g(p)+g_{1}(p) a^{\prime}$ if $g(p) \geqq a^{\prime}, p \notin C$ and $g^{+}(p)=a^{\prime}$ if $p \in C$ or $g(p)<a^{\prime}$. Let $\Delta g(p)$ be the gradient of $g$. Denote by $\Delta g(f(p))$ the directional derivative of a function $f$ at $p \in M$ along $\Delta g(p)$. The function $g^{+}$is smooth, and $\Delta g\left(g^{+}(p)\right)$ $=\left(1-g_{1}(p)\right) \Delta g(g(p))+\left(a^{\prime}-g(p)\right) \Delta g\left(g_{1}(p)\right)>0$ if $g_{1}(p)<1$. Let $b>a$ and $K=C \cup\{p \in M-C \mid g(p) \leqq b\}$. Replace $h, f$ by $g^{+}, f^{+}$respectively; then Proposition 2 yields the existence of a function $f^{+}$which approximates $g^{+}$on $K$. One has $f^{+}(p)<b^{\prime}$ for some $b^{\prime} \in \boldsymbol{R}$ and $p \in\left\{q \in M \mid g^{+}(q) \leqq b\right\}$. Let $g_{2}(p)=g_{a, b, g}(p)$ if $p \in M-C$. Since $g^{+}=g$ on $E=\{p \in M-C \mid a \leqq g(p) \leqq b\}$ it follows for the inner product $\left(\Delta g^{+}(p), \Delta g^{+}(p)\right) \geqq c^{\prime}>0$ if $p \in E$. Hence, since the gradient $\Delta f^{+}(p)$ approximates $\Delta g^{+}(p)$ (Proposition 2), $\left(\Delta g^{+}(p), \Delta f^{+}(p)\right)>0$ on $E$.

Define $f^{\prime}(p)=\left(1-g_{2}(p)\right)\left(g^{+}(p)+b^{\prime}-a\right)+g_{2}(p) f^{+}(p)$ if $p \in M-C$ and $f^{\prime}(p)=f^{+}(p)$ if $p \in C$. One has $\Delta g\left(f^{\prime}(p)\right)=\left(1-g_{2}(p)\right) \Delta g\left(g^{+}(p)\right)$ $+g_{2}(p) \Delta g\left(f^{+}(p)\right)+\left(-g^{+}(p)-b^{\prime}+a+f^{+}(p)\right) \Delta g\left(g_{2}(p)\right)>0$ if $p \in E$. The function $f^{\prime}$ is a critical finite function on $M$.

Corollary 1. The function $f^{\prime}$ of Theorem 2 coincides with some function $c \cdot L_{x}+d$ with $c>0$ on a subset of $M$ which contains all critical points of $f^{\prime}$.

Proof. The construction of $f^{+}$according to Proposition 2 is chosen such that $f^{+}$equals some function $c \cdot L_{x}+d$ on $M$ with $c>0$. Then $f^{\prime}=c \cdot L_{x}+d$ on the set $C \cup\{p \in M-C \mid g(p) \leqq a\}$.

By $[8$, pp. 353 and 383] there exists a critical finite function $f$ on $M$ with critical points $p_{1}, p_{2}, \cdots, p_{r}$ on $M$ such that $f\left(p_{1}\right)<f\left(p_{2}\right)$ $<\cdots<f\left(p_{r}\right)$ and the index $\lambda_{i}$ of $f$ at $p_{i}$ is less than $\operatorname{dim} M$.

Let $K$ be the union of the descending bowls $E_{i}$ associated with some critical point $p_{i}$ of $f$. More explicitly, let $p$ be a noncritical point of $f$; then $\phi(p)$ denotes the maximal orthogonal trajectory of $f$ through $p$, or if $p$ is a critical point of $f$ then $\phi(p)=\{p\}$. The set $E_{i}$ is defined as follows:

$$
E_{i}=\left\{p \in M \mid p_{i} \in \overline{\phi(p)}, f(p) \leqq f\left(p_{i}\right)\right\} .
$$

Let $\operatorname{dim} K=\max _{i=1}, \cdots, r \lambda_{i}$.

Corollary 2. $K$ is a deformation retract of $M$ and $\operatorname{dim} K \leqq$ $\operatorname{dim} M-1$.

Proof. The corollary of [5] yields the fact that $K$ is a deformation retract of $M$. Since $\lambda_{i} \leqq \operatorname{dim} M-1$ one has $\operatorname{dim} K \leqq \operatorname{dim} M-1$. 


\section{Strongly pseudoconvex Stein manifolds with $C^{2}$-boundary are smooth bounded.}

Proposition 3. A strongly pseudoconvex Stein manifold $M$ with $C^{2}$-boundary is smooth bounded.

Proof. From [3] and [4, p. 263] one has the existence of a strongly plurisubharmonic smooth function $h$ defined in a neighborhood $U$ of the boundary of $M$ such that $M \cap U=\{p \in M \mid h(p)<0\}$ and such that the gradient $\Delta h(p) \neq 0$ in $M \cap U$. Let $g(p)=-\log |h(p)|$. Since $A=M-U$ is compact it follows that $M$ is a smooth bounded manifold.

Theorem 3. Let $M$ be a strongly pseudoconvex Stein manifold with $C^{2}$-boundary of complex dimension $m$. There is a finite $\mathrm{CW}$-complex $K \subset M$ with real dimension $\leqq m$ such that $K$ is a deformation retract of $M$.

Proof. From Proposition 3 it follows that one can apply Theorem 2. Let $f^{\prime}$ be the critical finite function of Theorem 2. From [1] and Corollary 1 it follows that for each critical point of $f^{\prime}$ the index is less than or equal to $m$. Corollary 2 yields then the statement of Theorem 3.

\section{REFERENCES}

1. A. Andreotti and T. Frankel, The Lefschetz theorem on hyperplane sections, Ann. of Math (2) 69 (1959), 713-717.

2. C. Constantinescu and A. Cornea, Ideale Raender Riemannscher Flaechen, Ergebnisse der Math. und ihrer Grenzgebiete, Springer, Berlin, 1963.

3. H. Grauert, Ueber Modifikationen und exzeptionelle analytische Mengen, Math. Ann. 146 (1962), 331-368.

4. R. C. Gunning and H. Rossi, Analytic functions of several complex variables, Prentice-Hall, Englewood Cliffs, N. J., 1965.

5. G. Kalmbach, Deformation retracts and weak deformation retracts of noncompact manifolds, Proc. Amer. Math. Soc. 20 (1969), 539-544.

6. - Bowl functions on noncompact manifolds, Mimeographed Notes, Univ. of Illinois, Urbana, 1967.

7. J. Milnor, Morse theory, Ann. of Math. Studies, No. 51, Princeton Univ. Press, Princeton, N. J., 1963.

8. M. Morse, The existence of polar non-degenerate functions on differentiable manifolds, Ann. of Math. (2) 71 (1960), 352-383.

9. - "Bowls of a nondegenerate function on a compact differentiable manifold" in Differential and combinatorial topology, Princeton Univ. Press, Princeton, N. J., 1965, pp. 81-103.

10. J. H. C. Whitehead, Combinatorial homotopy. I, Bull. Amer. Math. Soc. 55 (1949), 213-245.

UNIVERSITY OF ILLINOIS 